\title{
Clinical reasoning and knowledge management in final year medical students: the role of Student-led Grand Rounds
}

\author{
This article was published in the following Dove Press journal: \\ Advances in Medical Education and Practice \\ 3 October 2017 \\ Number of times this article has been viewed
}

\section{David Arumaisingam \\ Kandiah}

Division of Psychiatry, Medical School, Faculty of Health and Medical Sciences, The University of Western Australia, Perth, WA, Australia
Correspondence: David Arumaisingam Kandiah

PO Box 1293, Bondi Junction, NSW

1355, Australia

Tel $+6 \mid 44880$ | $|4|$

Fax +6I $29034|24|$

Email davidkandiah@doctor.com
Objectives: The development of clinical reasoning and decision-making skills is often limited in medical school curricula. In reality, medical graduates acquire these skills during their first few years of residency. For many, this can be stressful as they may be working under limited supervision as a part of their rotations.

Methods: Student-led Grand Rounds was developed to transfer both explicit and tacit knowledge to final year medical students. This pilot project was to apply the principles of knowledge management to allow students to be exposed to the reasoning and decision making of common clinical presentations. Student feedback through questionnaires was collated at the end of the program.

Results: Based on feedback and focus groups, modifications were made to produce a stable program in subsequent clinical rotations. Formal feedback was collated from all the 76 students who participated in the first year. This represented $100 \%$ of the cohort for this clinical school for that year. There was a $100 \%$ response rate as the feedback forms were given and collected at the end of the last session per block. The student responses were both in ratings defined in a feedback forms and in written comments. A total of 74 of the 76 students rated the program highly. They enjoyed the nonthreatening interactions. The remaining two students preferred more didactic teaching.

Conclusion: This initiative allows an efficient transfer and utilization of knowledge. This could maximize the acquisition of practical knowledge by medical students as they finish their course in the transition to graduate medical practice.

Keywords: Student-led Grand Rounds, clinical reasoning, knowledge management, final year medicine, curriculum development

\section{Introduction}

Medical education in the 21st century is in a constant state of flux. Educational institutions are now investigating methods of improving the educational experiences of their graduates. This is through establishing initiatives that can maximize their competitive advantage and maintain the momentum of growth in student numbers and consumer satisfaction. This should be supported by formal evaluation processes that have become an inherent part of a university's assessment of their programs. This is also to attract the best possible students who would enhance the institution's reputation. Educational knowledge management (KM) in medical schools needs the creation of strategic communities. ${ }^{1}$ This is a natural evolution from clinical and research knowledge communities that already exist. 
Health professionals of any discipline are knowledge workers. Traditionally, they gather patient data and information, try and combine these data into a differential diagnoses based on the patterns they recognize from their earlier education and experiences. If they are well grounded with common presentations and can recognize patterns to plan immediate and ongoing patient care, they will be much more efficient in implementing patient management. Patient outcomes intuitively will be better. One of the traits of a skilled clinician at any level is the ability to create an appropriate shortlist of differential diagnoses, the most likely diagnosis, and plan treatment accordingly. The targeted education of junior clinicians has ramifications for health care delivery as there will be less speculative investigations ordered, less time spent in exposing the patient to more potentially dangerous tests, and more time spent caring for other patient needs. This should ultimately result in strategies for maximal patient care based on the best clinical guidelines and evidence-based practice. ${ }^{2}$

Decision making is implicit or explicit in every clinical activity encountered during undergraduate or graduate medical education, as it is fundamental to medical practice. Incorporating medical uncertainties and patient preferences could be a powerful unifying principle in medical training, integrating other components of the medical school curriculum. ${ }^{3}$ Decision-centered instruction would challenge learners to apply their knowledge of basic sciences and clinical medicine to address clinical decisions. ${ }^{3}$ Woods has reviewed work to suggest that biomedical knowledge can help learners develop a coherent and stable mental representation of disease categories. They are more likely to retain clinical knowledge over time and maintain diagnostic accuracy when faced with clinical challenges. Clinical teachers should be encouraged to make explicit connections between biomedical knowledge and clinical facts during medical training. ${ }^{4}$
An initiative to highlight this process is the development and implementation of Student-led Grand Rounds for final year medical students. The program was devised to address deficits identified from intern performance appraisals and focus groups of graduates from one Australian Medical School. The general consensus of the students, recent graduates, and their clinical supervisors was that they had a wide knowledge base but could not apply that efficiently in direct patient care.

\section{Methods}

Students in the largest clinical school at this university were assigned two specialties of their choice in their 8-week attachment to Internal Medicine (Selectives) in their final year. Students had been exposed to General Internal Medicine in the third year of their 4-year course and had a full clinical examination at the end of third year to assess their clinical skills. In the final year, they had a choice of two sub-specialty medical clinical terms of 4 weeks' duration each. There was previously little interaction between students to share their clinical experiences in their chosen disciplines, with their colleagues.

In this new initiative, students were given a chance to present in a formal setting, one patient that they have followed on the wards or in out-patients. The number of students per rotation ranged from 16 to 24 , so that some students missed out on presentation of a case, but all participated in the discussion after each case presentation. The topics were predetermined by the author (Table 1). This was based on common clinical presentations that they would have to manage as newly graduated junior doctors the following year. The students within the relevant rotations chose one patient with that clinical problem to present.

Table I Final year Internal Medicine Student-led Grand Rounds curriculum every wednesday (I-2 pm)

\begin{tabular}{|c|c|c|c|}
\hline Date & Topics & Date & Topics \\
\hline Block I & & Block 2 & \\
\hline \multirow[t]{3}{*}{ Week I } & Rheumatology & Week 5 & Infectious diseases \\
\hline & Polyarthritis & & Pyrexia of unknown origin \\
\hline & Immunological diseases, eg, SLE & & Pneumonia \\
\hline \multirow[t]{3}{*}{ Week 2} & Cardiology & Week 6 & Thoracic medicine \\
\hline & Acute coronary syndrome & & Carcinoma lung \\
\hline & Heart failure, eg, left ventricular failure & & Chronic obstructive pulmonary disease \\
\hline \multirow[t]{3}{*}{ Week 3} & Nephrology & Week 7 & Neurology \\
\hline & Acute kidney injury & & Stroke \\
\hline & Chronic kidney injury & & Seizures or peripheral neuropathy \\
\hline \multirow[t]{4}{*}{ Week 4} & Gastroenterology & Week 8 & Endocrinology \\
\hline & Peptic ulcer disease and GORD & & Diabetes mellitus - eg, diabetic \\
\hline & Acute or chronic liver disease & & Ketoacidosis \\
\hline & & & Thyroid diseases \\
\hline
\end{tabular}

Abbreviations: GORD, gastro-oesophageal reflux disease; SLE, systemic lupus erythematosus. 
Students discussed the patient and their approach to the clinical problems identified with the senior facilitator and relevant specialty registrar/consultant, prior to the actual presentation. All presentations were on PowerPoint with illustrative photos, electrocardiograms, radiological imaging, and other investigations relevant to the case presented. Each topic presentation lasted strictly for half an hour. The case was discussed for 20 minutes, and the last 10 minutes was spent discussing general principles in the management of that condition. The key emphasis in the final discussion was on practical management tips for junior doctors in preparation for when they assessed patients in the future on the wards, outpatient clinics, and the Emergency Department. The students shared their electronic presentations with their colleagues afterward. Generally, two facilitators were present for each case - a senior academic in Internal Medicine and a specialist in the discipline that the case was based.
At this stage, a Web-based knowledge repository of the student presentations has not been implemented as this educational initiative is site specific. The only portal that the students have access to is faculty based, and the medical school administrators wanted any student knowledge repository added to the portal only if it was a faculty-wide program. This initiative was extended to two of the nine other clinical schools 6 months later. Some students set up their own clinical rotation Facebook page and shared presentations on this site. Table 1 demonstrates the average 8 -week roster. The order can be changed based on availability of facilitators and student presenters.

A questionnaire was developed to gage student feedback as shown in Figure 1. These questionnaires were given to the students at the end of their last session as a part of their normal student evaluation process. The questionnaires were returned without their names to encourage honest feedback.

\section{Student-led Grand Rounds survey}

This survey is to evaluate Student Grand Rounds as a leaming tool in the final year of medical school. We would really appreciate you to take a few minutes and give some honest feedback to the questions below.

Firstly, could you indicate how much you agree or disagree with the statements below by circling the appropriate response (SA=strongly agree, $A=a g r e e, ~ N=n e u t r a l$, $\mathrm{D}=$ disagree, $\mathrm{SD}=$ strongly disagree):

1. I found Student Grand Rounds a worthwhile leaming expenience

SD D N A SA

2.I felt the number and length of sessions were suitable for my leaming.

SD D N A SA

2a. If you answered ' $D$ ' or 'SD' above, would you prefer more sessionsitime or less?

More Less

3. I felt the content was appropriate for my rotation (4th year Medical Specialties)

SD D N A SA

4. If an extra half-hour were made available after grand rounds with the facilitator to discuss leaming issues raised, I would find this useful.

SD D N A SA

S. Having the presentations available electronically after the sessions would further enhance my leaming.

SD D N A SA

For the next couple of questions could you circle which answer most closely matches your opinion:

6. What leaming method do you prefer?

Didactic Interactive Mixed

7. Do you prefer sessions nun solely by clinical teachers, or would you rather student-nun sessions with clinical teachers acting as facilitators and filling knowledge gaps? Teacher run Student run (Teacher facilitated)

8. Clinical Grand Rounds were set up to cover clinical topics that all junior doctors will face in patient care. Currently we have sections on the case history, key management issues and a discussion of the condition. In order to cover at least 14 topics per block, we have allocated only half an hour per topic. To maximize your leaming opportunities, you would prefer the facilitators to (circle appropriate letter):

A. Intempt regularly if there is a le aming issue

B. Allow the student presenter to finish each section and let them direct the questions.

C. Allow the student presenter to finish each section and let the facilitator direct the questions.

D. Let the presenter finish the whole topic and then lead with questions.

E. Have few slides and dissect the case history and leave the leaming on the case to each individual student in their own time.

Free text comments:

Finally, could you give feedback to the following two questions:

10. What aspects of grand rounds did you find useful and why?

11. What do you think could be improved and why?

Figure I Student-led Grand Rounds survey.

Notes: This survey is to evaluate Student-led Grand Rounds as a learning tool in the final year of medical school. We would really appreciate you to take a few minutes and give some honest feedback to the questions above. 
This was approved by the overall discipline coordinator of the Internal Medicine rotations.

\section{Results}

The feedback was collated from the 76 students who participated in one calendar year. There was a $100 \%$ response rate as the feedback forms were given and collected at the end of the last session per block. The relevant responses to the feedback from the students are graphically shown in Figure 2. All but two students found the initiative useful. The two students who were not satisfied wanted more didactic teaching. Many students desired notes of the sessions afterward to revise. At this stage, the students were asked to request presentations directly from their colleagues presenting. This worked well for this cohort of students, as they were happy to share their presentations. We also discussed issues of time management of the presentation, and this improved as the year progressed as the students were aware of the strict time restrictions. A prepresentation discussion with the senior facilitator reduced the inclusion of advanced material not compatible with the expected knowledge required at their level. The written feedback is shown in Box 1.

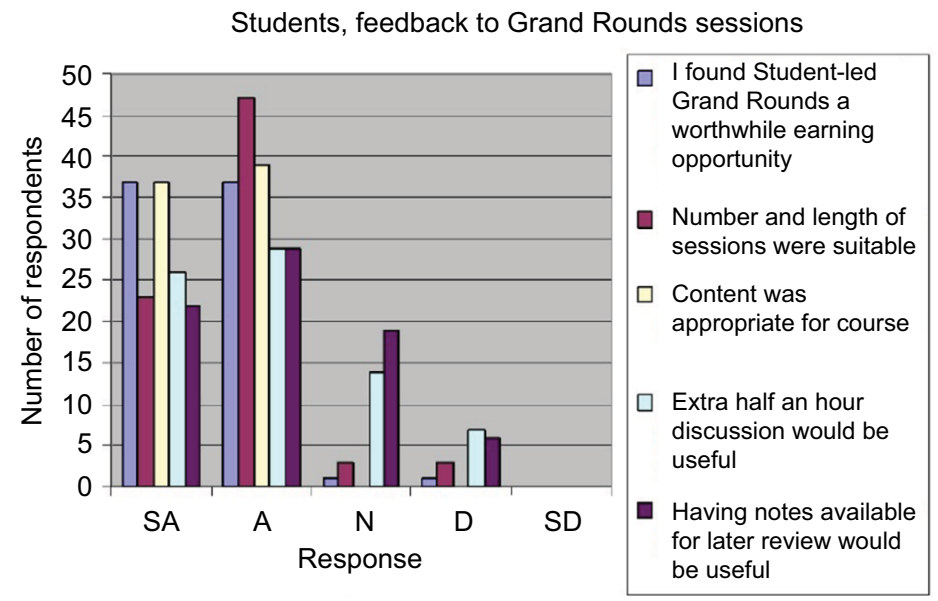

Figure 2 Student feedback.

Abbreviations: A, agree; D, disagree; N, neutral; SA, strongly agree; SD, strongly disagree.

\section{Box I Feedback on Student-led Grand Rounds}

- Really liked the interactive way of working through a case diagnosis, exam, investigation, management and physiology. This is particularly so when the case is one from an area that you're not presently posted. Good to get picked on by your mates and good to refresh. I think having a consultant present is neccessary for it to work. Having a registrar as well as a consultant to give out the "gold" is also very helpful. Much appreciated.

- Great student participation and excellent consultant feedback. One hour of solid, essential and relevant education! Should be continued for future terms.

- Clinical case oriented. Doctors also involved.

- Allows student interaction.

- Focused learning.

- Wide range of topics covered.

- Topics were common and important to have detailed knowledge of.

- Useful input from clinical teachers. Well prepared by all, go team!!

- Doing a presentation was invaluable.

- The chance to create and explore differential diagnosis as the case unfolds.

- Good general revision.

- Good practice for students in learning how to present. Interesting cases. Great consultant guidance.

- Encouraged to get involved.

- I loved the interaction and the opportunity for students to present.

- Two presentations per week is perfect - wish we could all have a chance to present.

- No negative feedback. We should have this in other disciplines like surgery.

- Would be good to include live patient demonstrations.

- If all consultants had such an active and enthusiastic interest in teaching, new graduates would be a lot better. 


\section{Discussion}

Kim et $\mathrm{al}^{5}$ reviewed 100 studies on developing teaching cases and synthesized 17 strategies around five core attributes of cases:

1. Relevant (to level of learner, goals and objectives, setting of cases);

2. Realistic (authentic, gradual disclosure of content);

3. Engaging (rich content. multiple perspectives, branching of content);

4. Challenging (difficulty, unusual cases, multiple cases);

5. Instructional (building on prior knowledge, assessment, feedback, and teaching aids).

In this educational initiative, all of these attributes were incorporated. Aligning KM in this educational initiative allowed the development of strategies based on the student feedback. These strategies allowed the following.

\section{Focus on long-term aims and outcomes}

Many of the activities of final year medical students can be aligned to the nurturing of students in their transition to junior doctors the following year. More than $95 \%$ of students who went through this program found this a worthwhile learning experience. While not everyone was satisfied, the clinical reasoning skills could be built on as a competitive advantage for the students in the workforce after graduation. This is especially relevant in the current workforce climate where there are now more graduates than there are clinical training positions.

Random feedback was sought the following year from these graduates and their supervisors. The comments of both groups were helpful as they were generally in the following two categories:

1. Improved readiness for supervised medical practice compared to the previous cohorts that did not have this educational activity.

2. Confidence and competence in caring for patients with these common clinical problems.

\section{Design for evolution}

This educational initiative allowed the incorporation of applied science in each presentation. This can further be used to catalyze growth and promotion of continuous learning within the student body. Most of the actual information and knowledge presented by the students was appropriate for their level, and all 76 students agreed or strongly agreed with this. This was ensured with prior prepresentation discussions with the facilitators.

\section{Development of community leaders}

The networking between students and their tutors, which is fundamental to this model of KM, facilitated positive interactions between these groups. Even the more timid students found the experience helpful, as their presentations allowed them to discuss the patients and their management with their supervising doctors beforehand. The consolidation of this knowledge was clearly evident as the students were able to focus on the key learning issues during their presentations. This has been shown before to be suitable for the retention of acquired knowledge. ${ }^{6,7}$

For knowledge networks to succeed, senior educators should recognize and cultivate an environment for knowledge sharing. Both space and time need to be given to knowledge workers to congregate and discuss ideas. Knowledge generation is often difficult to measure, and results of knowledge generation initiatives can take time to be successful. Political and social realities shape all forms of human conduct, and there may be reluctance to contribute or exchange knowledge if the prevailing culture does not support this. This network model is vulnerable to organizational culture. ${ }^{8}$ This facilitated initiative allowed these networks to be cultivated.

If an educational institution spans many sites, it will be imperative to develop and empower local leaders to build a good knowledge network model. This will certainly aid adoption of KM across the organization to improve the standardization of delivery of clinical education and training. Participation can be encouraged by

1. Organizing a mentoring program for upcoming junior clinicians.

2. Performance appraisals of supervisors, which should include a percentage for KM contributions.

3. Developing institution-wide repositories of knowledge.

4. The more junior staff members in the organization can be encouraged to contribute to KM if they can see it is the culture to do so.

5. Videoconferencing of sessions to smaller clinical school sites, where and when expert content clinicians were not available.

6. Creation of on-line resources to facilitate instruction and evaluation of knowledge that can be used for both formative and summative assessments. ${ }^{9}$

\section{Creation of individual value}

For community vitality to be promoted, there must be perceived and real value given to members recognizing their energy, personal time, and passion. Knowledge maps can allow members of a network to identify people to contact for 
specific knowledge and skills or techniques that are needed for successful completion of a task or project. When members are clear about the value from themselves and others, they often become more committed and active in their participation in group interactions. This was evident in this process as the students sought information from specific resources for their presentations after guidance from senior clinicians.

\section{Creation of a web of relationships}

Aligning KM with student learning enriched the relationships between students through direct and electronic communication. This enhanced deeper exchange of knowledge.

\section{Formalizing networks}

Knowledge works across networks and communities of practice. Nourishing and facilitating their functions are important if one wants to manage knowledge. Once the networks are functioning well on a human level then technology can be applied to ease the capture and sharing of knowledge across a network. ${ }^{10}$

Most current educational projects involve building electronic repositories of knowledge-structured documentbased knowledge, informal discussion-type knowledge, or repositories of who has the knowledge. The most important factor for a successful project is a fit with the organizational culture, leadership, and a connection to its educational values. In this example, Student-led Grand Rounds incorporated these ingredients allowing its successful implementation and spread within the organization.

Many teachers in professional educational programs have plenty of tacit knowledge. Some of this knowledge is difficult to make explicit. ${ }^{11}$ The common way to transfer or teach this knowledge is through long-term observation of people who have the knowledge. One has to careful of external influences in the transfer of knowledge, eg, by the pharmaceutical industry. Student-led Grand Rounds allowed a cost-effective method of transferring tacit knowledge through mentoring, supervision, and active personal communication of the clinical issues. There was always discussion on rational use of interventions whether pharmacological or interventional. ${ }^{12}$ This also facilitated intellectual curiosity based on discussions at the end of each presentation.

Educational research suggest that clinical teachers should stress the importance of both analytic (ie, conscious/controlled) and nonanalytic (unconscious/automatic) reasoning strategies. This can enable students to marshal reasoning processes in a flexible and context-specific manner. ${ }^{13}$

Norman stated that it had become evident that medical expertise lay in the availability of multiple representations of knowledge. He suggested that the critical element of learning may be in deliberate practice with multiple examples, facilitating the transfer of conceptual knowledge and add to a repository of already solved problems. ${ }^{14} \mathrm{KM}$ has an important part to play in medical practice of the 21 st century. Research needs to be ongoing for the best methods to apply KM tools and techniques to tertiary education and best professional practice. ${ }^{15}$ The pressures of health care delivery with outcome measures, determining best practice, maximizing reimbursement, and quality controls require accurate information and knowledge. Medical educational institutions should be encouraged to support educational initiatives that can be shown to improve the student learning experiences and quality of medical practice after graduation.

\section{Acknowledgment}

The author wishes to thank all the students and facilitators who participated in Student-led Grand Rounds.

\section{Author contributions}

DAK designed the program, was the senior facilitator, designed the questionnaire, collected the data, performed the data analysis, drafted the manuscript, and critically revised the article.

\section{Disclosure}

The author reports no conflicts of interest in this work.

\section{References}

1. Storck J, Hill PA. Knowledge diffusion through 'strategic communities'. Leadersh Organ Stud. 2000;41(2):63-74.

2. Kandiah DA. Insights into applied clinical learning: the 'Oscar' programme. SGH Proc. 2006;15(1):38-44.

3. Schwartz A. Medical decision making and medical education: challenges and opportunities. Perspect Biol Med. 2011;54(1):68-74.

4. Woods NN. Science is fundamental: the role of biomedical knowledge in clinical reasoning. Med Educ. 2007;41(12):1173-1177.

5. Kim S, Phillips WR, Pinsky L, Brock D, Phillips K, Keary J. A conceptual framework or developing teaching cases: a review and synthesis of the literature across disciplines. Med Educ. 2006;40(9):867-876.

6. Coles CR. Differences between conventional and problem-based curricula in their students' approaches to studying. Med Educ. 1985;19(4):308-309.

7. McManus IC, Richards P, Winder BC, Sproston KA. Clinical experience, performance in final examinations, and learning style in medical students: prospective study. Br Med J. 1998;316(128):345-350.

8. Davenport TH, Prusak L. Working Knowledge. Boston, USA: Harvard Business School Press; 2000.

9. Aronoff SC, Evans B, Fleece D, Lyons P, Kaplan L, Rojas R. Integrating evidence-based medicine into undergraduate medical education: combining on-line instruction with clinical clerkships. Teach Learn Med. 2010;22(3):219-223.

10. Davenport TH, De Long DW, Beers MC. Successful knowledge projects. Sloan Manage Rev. 1998;39(2):43-57.

11. Connell NAD, Klein JH, Powell PL. It's tacit knowledge but not as we know it: redirecting the search for knowledge. J Oper Res Soc. 2003;54: 140-152. 
12. Mather C, Fleising U, Taylor L. Translating knowledge from bench to bedside: the controversial social life of t-PA. Risk Manage. 2004;6(2): 49-60.

13. Eva KW. What every teacher needs to know about clinical reasoning. Med Educ. 2004;39(1):98-106.
14. Norman G. Research in clinical reasoning: past history and current trends. Med Educ. 2005;39(4):418-427.

15. De Camargo KR Jr. The thought style of physicians: strategies for keeping up with medical knowledge. Soc Stud Sci. 2002;32(5/6):827-855.

Advances in Medical Education and Practice

\section{Publish your work in this journal}

Advances in Medical Education and Practice is an international, peerreviewed, open access journal that aims to present and publish research on Medical Education covering medical, dental, nursing and allied health care professional education. The journal covers undergraduate education, postgraduate training and continuing medical education including emerging trends and innovative models linking education, research, and health care services. The manuscript management system is completely online and includes a very quick and fair peer-review system. Visit http://www.dovepress.com/testimonials.php to read real quotes from published authors.

Submit your manuscript here: http://www.dovepress.com/advances-in-medical-education-and-practice-journal 